\title{
Identification and Functional Validation of Differentially Expressed Micrornas in Ascites- derived Ovarian Cancer Cells Compared With Primary Tumour Tissue
}

\section{Yahui Jiang}

Shanghai Jiao Tong University Medical School Affiliated Ruijin Hospital

\section{Tianjiao Lyu}

Ruijin Hospital AmMed Cancer Center Shanghai

Hua Liu

Shanghai Jiao Tong University Medical School Affiliated Ruijin Hospital Lifei Shen

Shanghai Jiao Tong University Medical School Affiliated Ruijin Hospital

\section{Yiwen Shi}

Shanghai Jiao Tong University Medical School Affiliated Ruijin Hospital

Tianyu Zhou

Shanghai Jiao Tong University Medical School Affiliated Ruijin Hospital

Weiwei Feng ( $\square$ fww12066@rijh.com.cn )

Shanghai Jiao Tong University Medical School Affiliated Ruijin Hospital https://orcid.org/0000-00020685-9479

\section{Research}

Keywords: Ovarian cancer, Metastasis, Ascites-derived spheroids, Primary tumour tissue, microRNAs

Posted Date: August 3rd, 2020

DOI: https://doi.org/10.21203/rs.3.rs-51079/v1

License: (c) (1) This work is licensed under a Creative Commons Attribution 4.0 International License. Read Full License 


\section{Abstract}

Purpose: Ovarian cancer, manifested by malignant ascites, is the most lethal gynaecological cancer. Suspended ascites-derived spheroids may contribute to ovarian cancer metastasis. MicroRNAs (miRNAs) are also associated with ovarian cancer metastasis. Here, we aimed to investigate the differentially expressed miRNAs (DE-miRNAs) in ascites-derived spheroids compared with primary tumour tissue, which may regulate ovarian cancer metastasis.

Methods: The DE-miRNAs between ovarian cancer primary tumour tissues and ascites-derived spheroids were identified by GEO2R screening in dataset GSE65819. We used MiRTarBase and STRING to predict the target hub genes of DE-miRNAs and WebGestalt to perform functional analysis of hub genes. ALGGEN PROMO and TransmiR v2.0 were used to predict the common transcription factors (TFs) that potentially regulate DE-miRNAs expression. The observed differences in DE-miRNAs expression were validated with human ovarian cancer samples and ovarian cancer cell lines using PCR. The functions of DE-miRNAs on ovarian cancer progression were verified by transwell and angiogenesis assays.

Results: Through bioinformatics screening and experimental validation, miR-199a-3p, miR-199b-3p, miR199a-5p, miR-126-3p and miR-145-5p were identified as being significantly downregulated in ascitesderived spheroids compared with primary tumour tissues. In addition, TFAP2A was identified as a potentially common upstream TF regulating the expression of the abovementioned DE-miRNAs. The overexpression of miR-199a-3p, miR-199b-3p, miR-199a-5p could inhibit ovarian cancer invasion, and the overexpression of miR-145-5p could inhibit angiogenesis.

Conclusion: The downregulated expression of miR-199a-3p, miR-199b-3p, miR-199a-5p, miR-126-3p and miR-145-5p in ascites-derived spheroids plays a key role in promoting ovarian cancer progression, which may represent novel molecules for targeted therapy for ovarian cancer.

\section{Introduction}

Ovarian cancer has become the most lethal gynaecological cancer worldwide. Since there is no obvious clinical presentation in the early phase, ovarian cancer patients are mostly diagnosed in the late stages, presenting with ascites accumulation in the abdominal cavity. The presence of malignant ascites correlates with a deterioration in the quality of life and poor prognosis(1). The ovarian cancer cells are shed from the primary tumour site, subsequently becoming suspended in ascites and forming spheroids. Finally, the spheroids, which are highly invasive, adhere to the peritoneum and form a new metastasis tumour site. Thus, spheroids appear to play a key role in ovarian cancer implantation metastasis, and the unique expression profile of suspended ascites-derived tumour cells deserves intensive study.

microRNAs (miRNAs) are endogenously expressed, small (19-25 nucleotides), noncoding RNAs that are cleaved from 70-100 nucleotide hairpin precursors called pre-miRNAs. Once the pre-miRNAs reach the cytoplasm, under the actions of various enzymatic reactions, they are cleaved into mature miRNAs that downregulate the expression of their targets by inhibiting mRNA translation or promoting mRNAs 
degradation. miRNAs are estimated to control over $50 \%$ of the activities of all protein coding genes and are unsurprisingly involved in ovarian cancer oncogenesis(2). miRNAs can act as potential biomarkers, diagnostic tools and therapeutic targets of ovarian cancer. For instance, the upregulation of miR-92, miR21 and miR-19a has been used as a sign of ovarian cancer, and cancer cell-secreted microvesicles contain elevated levels of miR-21, which induces myoblast apoptosis and lead to cancer cachexia(3).

Bioinformatics approaches have become an effective and systematic means of screening potential target molecules, and the Gene Expression Omnibus (GEO) database harbours a vast amount of gene expression profiles that are useful in distinguishing differentially expressed genes(4). In our study, to identify the differentially expressed miRNAs (DE-miRNAs) between ovarian cancer primary tumour tissue and ascites-derived cancer cells, we screened the miRNA expression profile of the microarray dataset GSE65819. The potential downstream target genes of DE-miRNAs were predicted using miRTarBase, and their biological significances were enriched by gene ontology (GO) annotation and Kyoto Encyclopedia of Gene and Genomes (KEGG) pathway analysis. By drawing a protein-protein interaction (PPI) network, we identified the hub target genes. Subsequently, we used human ovarian cancer samples and ovarian cancer cell lines to verify the results of bioinformatic analysis. The goal of this study was to identify miRNAs that may promote the metastasis of ovarian cancer ascites-derived cancer cells and to further investigate their potential molecular mechanisms by bioinformatic analysis and experimental validation.

\section{Methods And Materials}

\section{Selection of microarray data from GEO database}

To analyse the differential expression of miRNAs between ovarian cancer primary tumour and ascitesderived tumour cells, we screened GEO datasets and selected the microarray dataset GSE65819 for further study. This dataset is based on the GPL19765 platform (NanoString nCounter miRNA Human v2.1), which contains 121 high-grade epithelial ovarian cancer samples. After checking the basic information (including case ID, sample point and sample type) for each sample, we chose data from 3 patients' matched primary tumour and ascites-derived tumour cells, which were collected as soon as the patients were diagnosed without any therapy.

\section{Screen for DE-miRNAs}

GEO2R (http://www.ncbi.nlm.nih.gov/geo/geo2r/) can be used to perform modern R language analyses of GEO information under the same trial conditions(4). Therefore, GEO2R was used to screen the DEmiRNAs between primary tumours and ascites-derived tumour cells of ovarian cancer patients. $P$-value $<0.05$ and |fold change (FC)| 2 were set as the criteria for identifying DE-miRNAs.

\section{Prediction of target genes}

MiRTarBase (http://mirtarbase.mbc.nctu.edu.tw/php/index.php) is an experimentally validated miRNAtarget interactions database(5) We used miRTarBase to predict the target downstream genes of the top 
six most downregulated miRNAs.

\section{Analysis of GO and KEGG pathway}

WebGestalt (http://www.webgestalt.org) is one of the most widely used gene set enrichment analysis tools that allows users extract biological insights from genes of interest.(6) WebGestalt was used to perform functional and pathway enrichment of the predicted target genes of the six most downregulated miRNAs, including GO and KEGG pathway analysis.

\section{Construction of the PPI network and DE-miRNA-hub gene network}

The STRING database (http://string-db.org) provides uniquely comprehensive coverage and ease of access to both experimental and predicted protein interaction information.(7) We used the STRING database to obtain functional associations among the target genes of DE-miRNAs. To identify the hub genes of DE-miRNAs, the Cytoscape (3.7.0) plugin cytohubba was used to calculate the degree of each target gene. The top 20 target genes were identified as hub genes, and the DE-miRNA-hub gene network was established using Cytoscape.

\section{Prediction of transcription factors of DE-miRNAs}

The potential transcription factors of DE-miRNAs were predicted using ALGGEN PROMO (http://alggen.Isi.upc.es) and TransmiR v2.0 (http://www.cuilab.cn/transmir). $(8,9)$ Then, we took the intersection of the results obtained from ALGGEN PROMO and TransmiR v2.0 and drew a VENN diagram using a tool from bioinformatics and evolutionary genomics (http://bioinformatics.psb.ugent.be/webtools/Venn).

\section{cDNA synthesis and quantitative polymerase chain reaction (qPCR)}

TRIzol reagent (Invitrogen) was used to extract total RNA from cells from the different treatments. Reverse transcription was performed using a BioTNT microRNA reverse transcription kit (BioTNT, Shanghai, China), and the primers used for reverse transcription of miRNAs were also synthesized by BioTNT Co. Ltd. qPCR was performed using a BioTNT microRNA Real-Time PCR PreMIX kit and a qPCR system (QuantStudio TM 6 Flex, Thermo-ABI). The miRNAs expression levels were calculated by the $\Delta \Delta \mathrm{Ct}$ method. The sequences of the miRNA primers are listed in Supplemental Table 1.

\section{Human samples collection}

The primary tumour tissues and ascites were harvested from patients who were diagnosed with highgrade serous ovarian cancer in 2017-2019 in the Department of Gynaecology and Obstetrics, Ruijin Hospital. According to the inclusion criteria, (histologically diagnosed to be high-grade serous ovarian cancer combined with obvious ascites and no treatment before receiving tumour reductive surgery), tissue was collected from 12 patients for further experiments. The average age of these 12 patients were 55. The tumour stage of 5 patients were IIIc, and the other 7 patients were IV. All experiments were 
approved by the Ethics Committee of Ruijin Hospital, Shanghai Jiao Tong University, School of Medicine, and informed consent was obtained from all patients.

\section{Cell lines and culture conditions}

The human ovarian cancer cell line HEY was obtained from the laboratory of Dr Robert Bast at the University of Texas MD Anderson Cancer Center, Houston, TX. The human ovarian cancer cell lines OVCAR3 and SKOV3 were purchased from ATCC and the Chinese Academy of Sciences Cell Bank, respectively. The human ovarian cancer cell lines A2780 and OVCA433 were obtained from the Shanghai Key Laboratory of Female Reproduction Endocrine Related Disease, Obstetrics and Gynaecology Hospital, Fudan University. Human umbilical vein endothelial cells (HUVECs) was purchased from ScienCell.

For adherent cultures (2D cultures), cells were cultured in 100-mm TC-treated Culture Dishes (430167, Corning) with RPMI 1640 medium containing 10\% FBS, $100 \mathrm{U} / \mathrm{ml}$ penicillin and $100 \mu \mathrm{g} / \mathrm{ml}$ streptomycin at $37^{\circ} \mathrm{C}$ under an atmosphere with $5 \% \mathrm{CO}_{2}$.

For suspended cultures (3D cultures), cells were cultured in 100-mm Ultra-Low Attachment Culture Dishes (3262, Corning) with RPMI 1640 medium containing 10\% FBS, $100 \mathrm{U} / \mathrm{ml}$ penicillin and $100 \mu \mathrm{g} / \mathrm{ml}$ streptomycin at $37^{\circ} \mathrm{C}$ under an atmosphere with $5 \% \mathrm{CO}_{2}$.

HUVECs were cultured in ECM medium (ScienCell) containing 5\% FBS, $100 \mathrm{U} / \mathrm{ml}$ penicillin, $100 \mu \mathrm{g} / \mathrm{ml}$ streptomycin and $1 \% \mathrm{ECGS}$ at $37^{\circ} \mathrm{C}$ under an atmosphere with $5 \% \mathrm{CO}_{2}$.

\section{MiRNA overexpression}

The miRNA mimics and negative control were purchase from RiboBio (Guangzhou, China). We transfected the miRNA mimics and negative control into HEY cell lines with Lipofectamine ${ }^{T M} 3000$ according to the manufacturer's protocol and verified the overexpression efficiency by qPCR.

\section{Transwell invasion assay}

The Transwell system (24-well insert; pore size $8 \mathrm{~mm}$; Corning Costar) was used to measure the invasiveness of the HEY cell lines. The inserts were coated with $50 \mu \mathrm{l}$ of Matrigel (BD Biosciences Pharmingen), and $3 \times 10^{4}$ cells suspended in $0.2 \mathrm{ml}$ of fresh medium without FBS were added to the upper well of the chamber. Then, $600 \mu \mathrm{l}$ of complete medium with $10 \%$ FBS was added to the lower well. After incubating for $16 \mathrm{~h}$, the cells on the upper surface of the membrane were swiped off with cotton swabs, and the cells adhering to the lower surface of the inserts were fixed and stained with haematoxylin. The number of stained cells in five representative fields were randomly counted for each insert using an Olympus light microscope at 100x magnification.

\section{Angiogenesis assay}


HUVECs were treated with serum starvation medium for $6 \mathrm{~h}$ before performing angiogenesis assay. Matrigel $(300 \mu \mathrm{l})$ was added to each well of a 24 -well plate, and the plate was then transferred to a $37^{\circ} \mathrm{C}$ incubator for 30 mins. HUVECs were trypsinized and resuspended into $5 \times 10^{5} / \mathrm{ml}$ with serum-free ECM, and then $200 \mu \mathrm{l}$ of each cell suspension was added into the Matrigel-coated wells. Finally, the HUVECs were incubated in the conditioned medium from ovarian cancer cells with different treatments for 4-6 $\mathrm{h}$. Subsequently, images were taken under a microscope and analysed using ImageJ.

\section{Statistical analysis}

Differences between two groups were analysed by unpaired Student's t-test with SPSS and GraphPad Prism 8 . We considered $p$ value $<0.05$ as significant.

\section{Results}

\section{Screening DE-miRNAs between ovarian cancer primary tumours and ascites-derived tumour cells}

The microRNA array GSE65819 contains 114 samples collected from 92 high-grade serous ovarian cancer (HGSOC) patients at different time points. The sample types included primary tumour tissue, ascites-derived tumour cells and metastasis tumour tissue. We selected the microRNA array results of the primary tumour tissue and ascites-derived tumour cells harvested from $3 \mathrm{HGSOC}$ patients at the first time they were diagnosed with HGSOC (Fig.1A). Using GEO2R to analyse the DE-miRNAs from the 3 pairs of primary tumour tissue and ascites-derived tumour cells, we identified 69 DE-miRNAs, among which 68 were downregulated and only miR-1245a was upregulated in ascites-derived tumour cells. MiR-199a-3p, miR-199b-3p, miR-199a-5p, miR-145-5p, miR-126-3p and miR-508-5p were the top 6 most downregulated miRNA in ascites-derived tumour cells compared with the primary tumour tissues(Fig.1B,C).

\section{Prediction of target genes of DE-miRNAs and pathway enrichment analysis}

We used miRTarBase to predict the target downstream genes of miR-199a-3p, miR-199b-3p, miR-199a-5p, miR-145-5p, miR-126-3p and miR-508-5p, resulting in the identification of 1273 probable target genes. After KEGG pathway analysis, we observed that the target genes of DE-miRNAs were primarily enriched in "pancreatic cancer", "chronic myeloid leukaemia" and "focal adhesion". Regarding the GO analysis, "positive regulation of cell migration", "positive regulation of cellular component movement" and "vasculature development" were the most significant terms in biological process (BP); "transferase complex, transferring phosphorus containing groups", "nuclear transcription factor complex" and "transcription factor complex" were the most significant terms in cellular component (CC); and "protein kinase activity", phosphotransferase activity, alcohol group as acceptor" and "kinase activity" were the most significant terms in molecular function (MF)(Fig.2B-E).

\section{Identification of hub target genes of DE-miRNAs}


To identify the hub target genes of DE-miRNAs, a PPI network was evaluated by using the STRING database and visualized with Cytoscape. Considering the degree of the target genes, AKT1, MYC, EGFR, VEGFA, CDH1, MAPK1, KRAS, ESR1, MAPK8, UBC, IGF1, ERBB2, MTOR. SIRT1, ACTB, CD44, FGF2, SMAD4, MAPK14 and SMAD2 were top 20 genes with the highest degree values and were identified as hub genes of DE-miRNAs. From the PPI network shown in Fig.2A, it was obvious that miR-199a-3p, miR199b-3p, miR-199a-5p, miR-145-5p and miR-126-3p regulate the expression of multiple hub genes and can also collectively regulate the expression of a single gene. However, miR-508-5p can only regulate the expression of UBC and is not associated with the other identified DE-miRNAs. Therefore, we chose miR199a-3p, miR-199b-3p, miR-199a-5p, miR-145-5p and miR-126-3p for further study.

\section{Identification of potential upstream transcription factors of DE-miRNAs}

miRNAs regulate the expression of their target genes, but the expression of miRNAs themselves can also be regulated by transcription factors (TFs). miR-199a-1, miR-199a-2, miR-199b, miR-145 and miR-126 are the pre-miRNAs of miR-199a-3p, miR-199b-3p, miR-199a-5p, miR-145-5p and miR-126-3p. We used ALGGEN PROMO and TransmiR v2.0 to predict the probable transcription factors that regulate the 5 premiRNAs mentioned above. Twenty-six and 6 TFs were identified as potential regulators of all 5 premiRNAs using ALGGEN PROMO and TransmiR v2.0, respectively (Fig.3A, B). After taking the intersection, we observed that TFAP2A was the common TF that may regulate all 5 pre-miRNAs mentioned above and further affect the expression of miR-199a-3p, miR-199b-3p, miR-199a-5p, miR-145-5p and miR-126-3p (Fig.3C).

\section{Verification of DE-miRNA expression differences between primary tumours and ascites-derived tumour cells using ovarian cancer patient samples and ovarian cell lines}

We collected primary tumours and ascites-derived tumour cells from 12 ovarian cancer patients from 2017 to 2019. After evaluating the expression levels of miR-199a-3p, miR-199b-3p, miR-199a-5p, miR-145$5 p$ and miR-126-3p, we observed that the expression of miR-199a-3p, miR-199b-3p, miR-199a-5p and miR$145-5 p$ was lower in ascites-derived tumour cells than in the primary tumour $(p<0.01)$. The ascites-derived tumour cells from 11 of 12 patients had a lower miR-199b-3p expression level than that of the primary tumour in $(p<0.01)$ (Fig.4A).

To investigate whether this phenomenon could also be observed using ovarian cancer cell line models, we use 2D and 3D cultures to mimic the growth states of primary tumours ascites-derived tumour cells, respectively. The results showed that compared with the 2D culture, miR-199a-3p, miR-199b-3p, miR199a-5p, miR-145-5p and miR-126-3p were decreased in the 3D-cultured HEY cell line. The expression of miR-199a-3p, miR-199b-3p, miR-145-5p and miR-126-3p but not miR-199a-5p was decreased in the 3Dcultured A2780 cell line. For 3D-cultured OVCA433, SKOV3 and OVCAR3 cells, most of 5 assayed DEmiRNAs were not downregulated compared with the 2D cultures, and some of the DE-miRNAs were even upregulated under 3D culture conditions (Fig.4B). 


\section{Effects of miR-199a-3p, miR-199b-3p, miR-199a-5p, miR-145-5p and miRNA-126-3p on invasion and angiogenesis}

The pathway analysis results revealed that the dominant functions of the identified DE-miRNAs were migration and vasculature development. Therefore, we used Transwell invasion and angiogenesis assays to investigate the effects of the 3D-cultured HEY cell line. After overexpressing miR-199a-3p, miR-199b-3p and miR-199a-5p, the invasion ability of 3D-cultured HEY cells were inhibited. The angiogenesis of HUVECs was abrogated when miR-145-5p was overexpressed in 3D-cultured HEY cells. In summary, miR199a-3p, miR-199b-3p and miR-199a-5p can inhibit spheroid ovarian cancer cell invasion, while miR-145$5 p$ can inhibit spheroid ovarian cancer cell angiogenesis.(Fig.5A, B).

\section{Discussion}

The primary metastatic route of ovarian cancer is peritoneal dissemination, which is responsible for the greatest morbidity and mortality in ovarian cancer patients. Tumour cells shed from the primary tumour and become suspended in ascites to implant in the peritoneum and form new metastasis tumour sites. Thus, shed tumour cells carried in ascites are important for metastasis process. MicroRNAs are small, highly conserved noncoding RNA molecules that play an important role in ovarian cancer metastasis and are considered to be potential biomarkers for monitoring ovarian cancer(10). However, little research had focused on miRNA expression profile variations in ovarian cancer cells from ascites shed from primary tumour sites. Therefore, the goal of the present study was to elucidate the dominant differential miRNAs in ascites-derived tumour cells compared with the primary tumour sites and determine the contribution of these miRNAs to ovarian cancer metastasis.

The results showed that the expression changes of miRNAs in ascites-derived tumour cells primarily involved their downregulation, with miR-199a-3p, miR-199b-3p, miR-199a-5p, miR-145-5p and miR-126-3p exhibiting the most significant changes. Through the functional analysis of these miRNAs, we observed that they may be involved in the migration of ascites-derived tumour cells and promoting angiogenesis. Several studies have investigated the relationship of the above miRNAs with ovarian cancer. Lower miR199a expression was shown to be significantly correlated with a poor prognosis of ovarian cancer(11). The mature miRNAs miR-199a, miR-199a-3p and miR-199a-5p can also manipulate ovarian cancer development. Most studies of miR-199a-3p have shown that it can enhance the cisplatin sensitivity of ovarian cancer cells via a unique mechanism, such as targeting ITGB8(12) or DDR1(13). MiR-199a-3p and miR-199a-5p were also demonstrated to be ovarian cancer suppressors by inhibiting cancer cell proliferation, adhesion and invasion(14, 15). MiR-199a-3p was also shown to inhibit ovarian cancer proliferation and invasion by targeting PLXNB2(16). The interaction network of noncoding RNAs is essential for tumour progression. Several noncoding RNAs, such as CircMUC16(17), IncRNA LUCAT1(18) and IncRNA NORAD(19) promote ovarian cancer progression through miR-199a-3p and miR-199a-5p. Pan et al reported that miR-126 was underrepresented in exosomes from the plasma of epithelial ovarian cancer patients compared with that of healthy women(20). MiR-145 was shown to serve as an outstanding biomarker of ovarian cancer, since it is significantly downregulated in the sera of ovarian 
cancer patients compared to healthy women(21). It was also reported that miR-145 can suppress ovarian cancer cell growth and invasion by targeting CCD2, E2F3(22) and TRIM2(23). Many noncoding RNAs such as IncRNA-ROR(24) and circ-ITCH(25) play a role in regulating ovarian cancer though miR-145. However, little research has been performed on miR-145-5p, which is its mature miRNA. Our results showed that miR-199a-3p, miR-199a-5p and miR-199b-3p can suppress the invasion of spheroid ovarian cancer cells and that miR-145-5p can inhibit the angiogenesis of HUVECs induced by suspended ovarian cancer cells, which had not been previously reported.

Since miR-199a-3p, miR-199b-3p, miR-199a-5p, miR-145-5p and miR-126-3p were significantly downregulated in ascites-derived ovarian cancer tumour cells, the mechanism associated with this phenomenon was intriguing. The level of mature miRNA expression is associated with that of its precursor. For example, the mature miRNAs miR-199a-3p and miR-199a-5p are formed after cleavage from their precursor pre-miR-199a (one in chromosome 1 called miR-199a-2, another in chromosome 19 called miR-199a-1)(26). Therefore, we predicted the probable upstream transcription factors that could potentially regulate the pre-miRNAs of DE-miRNAs. The results showed that TFAP2A may be the common TF that can control the expression of all the pre-miRNAs of the identified DE-miRNAs. However, the ability of TFAP2A to regulate the abovementioned pre-miRNAs has yet to be reported and deserves further study. According to the results of previous studies, TFAP2A is significantly upregulated in ovarian cancer tissue compared to normal ovarian epithelial tissue(27). Furthermore, clinical findings have shown that TFAP2A favours the survival of ovarian cancer patients, with a potential mechanism being that TFAP2A may suppress ovarian cancer invasion and peritoneal carcinomatosis(28).

Using 12 pairs of primary tumour tissues and ascites-derived tumour cells collected from ovarian cancer patients to validate the observed expression differences in the identified DE-miRNAs, we showed that miR-199a-3p, miR-199b-3p, miR-199a-5p, miR-126-3p and miR-145-5p were significantly decreased in suspended ascites-derived tumour cells compared with primary tumour tissues, supporting the bioinformatics screening results. We performed adherent and suspended cultivation of ovarian cancer cells lines to mimic the growth environment characteristics of primary tumour tissue and ascites-derived tumour cells to further validate the decreased expression of the identified DE-miRNAs when ovarian tumour cells are suspended in ascites. However, only the HEY and A2780 cell lines exhibited decreased levels of the identified DE-miRNA expression in 3D culture. The expression of these DE-miRNAs in other assayed ovarian cell lines (OVCA433, SKOV3 and OVCAR3) remained unchanged or even increased in 3D cultures compared with that observed in 2D cultures, which did not match the bioinformatics screening and patient sample validation results. Therefore, we presume that the tumour microenvironment plays a large role in DE-miRNA expression changes when ovarian cancer cells shed from the primary tumour and become suspended in ascites, since the survival of ovarian cancer cells depends on a number of other components, such as stromal cells and immune cells in the tumour microenvironment. The mechanism associated with this process requires further in-depth study.

In summary, in the present study, we confirmed that the expression of miR-199a-3p, miR-199b-3p, miR199a-5p, miR-126-3p and miR-145-5p was reduced in ascites-derived tumour cells compared with primary 
tumour tissues, which may contribute to ovarian cancer peritoneal metastasis by promoting invasion and angiogenesis. TFAP2A was predicted to be a common transcription factor of the above 5 miRNAs, and further studies should be performed to verify its associated regulatory function and mechanism. In addition, the tumour microenvironment might give rise to the aberrant expression of miRNAs in ascitesderived tumour cells, but the underlying mechanisms remain to be elucidated.

\section{Conclusion}

In summary, miR-199a-3p, miR-199b-3p, miR-199a-5p, miR-126-3p and miR-145-5p were significantly downregulated in ascites-derived ovarian cancer cells compared with primary tumour tissues. TFAP2A may be a common upstream TF that can regulate the expression of the identified DE-miRNAs. The results of the present study suggest that decreased miR-199a-3p, miR-199b-3p and miR-199a-5p may expression promote the invasion of suspended ascites-derived tumour cells. The deregulation of miR-145-5p in suspended ascites-derived tumour cells may promote angiogenesis. Further study on the identified DEmiRNAs and their functions could be useful in the treatment of ovarian cancer.

\section{Declarations}

\section{Funding:}

This work was supported by the Shanghai Municipal Education Commission-Gaofeng Clinical Medicine Grant (Grant No. 20172003), and Guangci Distinguished Young Scholars Training Program of Shanghai Jiaotong University School of Medicine affiliated Ruijin Hospital (GCQN-2019-B12).

\section{Conflicts of interest/Competing interests:}

The authors declare that there is no conflict of interest.

\section{Authors` contributions:}

All authors contributed to the study conception and implement. Jiang Yahui was contributed to write the manuscript and conduct the experiments. Lyu Tianjiao was contributed to write the manuscript and did the data analysis. Liu Hua and Shen Lifei collected all the human ovarian cancer samples for experimental validation. Shi Yiwen and Zhou Tianyu helped to conduct part of the experiments. Feng Weiwei designed this study and was the corresponding author of this manuscript.

Availability of data and materials: The raw data supporting the conclusions of this manuscript will be made available by the authors, without undue reservation, to any qualified researcher.

Consent to participate: The informed consents were obtained from all patients included in the study.

Consent for publication: The participant has consented to the submission of the article to the journal. 
Ethics approval: All experiments were approved by the Ethics Committee of Ruijin Hospital, Shanghai Jiao Tong University, School of Medicine.

\section{Acknowledgements:}

We would like to thank all the patients that agreed with dedicating their ovarian cancer samples. We wish them good health. In addition, we appreciated to all the efforts that was made by the surgeons in our department. And we would like to express our gratitude to the support given by Shanghai Municipal Education Commission-Gaofeng Clinical Medicine Grant (Grant No. 20172003) and Guangci Distinguished Young Scholars Training Program of Shanghai Jiaotong University School of Medicine affiliated Ruijin Hospital (GCQN-2019-B12).

\section{References}

1. Kim S, Kim B, Song YS. Ascites modulates cancer cell behavior, contributing to tumor heterogeneity in ovarian cancer. Cancer science. 2016;107(9):1173-8.

2. Deb B, Uddin A, Chakraborty S. miRNAs and ovarian cancer: An overview. Journal of cellular physiology. 2018;233(5):3846-54.

3. He WA, Calore F, Londhe P, Canella A, Guttridge DC, Croce CM. Microvesicles containing miRNAs promote muscle cell death in cancer cachexia via TLR7. Proceedings of the National Academy of Sciences of the United States of America. 2014;111(12):4525-9.

4. Barrett T, Wilhite SE, Ledoux P, Evangelista C, Kim IF, Tomashevsky M, et al. NCBI GEO: archive for functional genomics data sets-update. Nucleic acids research. 2013;41(Database issue):D991-5.

5. Chou CH, Shrestha S, Yang CD, Chang NW, Lin YL, Liao KW, et al. miRTarBase update 2018: a resource for experimentally validated microRNA-target interactions. Nucleic acids research. 2018;46(D1):D296-d302.

6. Liao Y, Wang J, Jaehnig EJ, Shi Z, Zhang B. WebGestalt 2019: gene set analysis toolkit with revamped Uls and APIs. Nucleic acids research. 2019;47(W1):W199-w205.

7. Szklarczyk D, Franceschini A, Kuhn M, Simonovic M, Roth A, Minguez P, et al. The STRING database in 2011: functional interaction networks of proteins, globally integrated and scored. Nucleic acids research. 2011;39(Database issue):D561-8.

8. Tong Z, Cui Q, Wang J, Zhou Y. TransmiR v2.0: an updated transcription factor-microRNA regulation database. Nucleic acids research. 2019;47(D1):D253-d8.

9. Farré $D$, Roset $R$, Huerta $M$, Adsuara JE, Roselló $L$, Albà $M M$, et al. Identification of patterns in biological sequences at the ALGGEN server: PROMO and MALGEN. Nucleic acids research. 2003;31(13):3651-3.

10. Staicu CE, Predescu DV, Rusu CM, Radu BM, Cretoiu D, Suciu N, et al. Role of microRNAs as Clinical Cancer Biomarkers for Ovarian Cancer: A Short Overview. Cells. 2020;9(1). 
11. Nam EJ, Yoon H, Kim SW, Kim H, Kim YT, Kim JH, et al. MicroRNA expression profiles in serous ovarian carcinoma. Clinical cancer research : an official journal of the American Association for Cancer Research. 2008;14(9):2690-5.

12. Cui Y, Wu F, Tian D, Wang T, Lu T, Huang X, et al. miR-199a-3p enhances cisplatin sensitivity of ovarian cancer cells by targeting ITGB8. Oncology reports. 2018;39(4):1649-57.

13. Deng Y, Zhao F, Hui L, Li X, Zhang D, Lin W, et al. Suppressing miR-199a-3p by promoter methylation contributes to tumor aggressiveness and cisplatin resistance of ovarian cancer through promoting DDR1 expression. Journal of ovarian research. 2017;10(1):50.

14. Kinose Y, Sawada K, Nakamura K, Sawada I, Toda A, Nakatsuka E, et al. The hypoxia-related microRNA miR-199a-3p displays tumor suppressor functions in ovarian carcinoma. Oncotarget. 2015;6(13):11342-56.

15. Liu X, Yao B, Wu Z. miRNA-199a-5p suppresses proliferation and invasion by directly targeting NFKB1 in human ovarian cancer cells. Oncology letters. 2018;16(4):4543-50.

16. Xiang G, Cheng Y. MiR-126-3p inhibits ovarian cancer proliferation and invasion via targeting PLXNB2. Reproductive biology. 2018;18(3):218-24.

17. Gan X, Zhu H, Jiang X, Obiegbusi SC, Yong M, Long X, et al. CircMUC16 promotes autophagy of epithelial ovarian cancer via interaction with ATG13 and miR-199a. Mol Cancer. 2020;19(1):45.

18. Liu HZ, Liu GY, Pang WW, Zhang H, Zeng ZJ, Wang HJ. LncRNA LUCAT1 promotes proliferation of ovarian cancer cells by regulating miR-199a-5p expression. European review for medical and pharmacological sciences. 2020;24(4):1682-7.

19. Xu C, Zhu LX, Sun DM, Yao H, Han DX. Regulatory mechanism of IncRNA NORAD on proliferation and invasion of ovarian cancer cells through miR-199a-3p. European review for medical and pharmacological sciences. 2020;24(4):1672-81.

20. Pan C, Stevic I, Müller V, Ni Q, Oliveira-Ferrer L, Pantel K, et al. Exosomal microRNAs as tumor markers in epithelial ovarian cancer. Molecular oncology. 2018;12(11):1935-48.

21. Liang H, Jiang Z, Xie G, Lu Y. Serum microRNA-145 as a novel biomarker in human ovarian cancer. Tumour biology : the journal of the International Society for Oncodevelopmental Biology and Medicine. 2015;36(7):5305-13.

22. Hua M, Qin Y, Sheng M, Cui X, Chen W, Zhong J, et al. miR-145 suppresses ovarian cancer progression via modulation of cell growth and invasion by targeting CCND2 and E2F3. Molecular medicine reports. 2019;19(5):3575-83.

23. Chen X, Dong C, Law PT, Chan MT, Su Z, Wang S, et al. MicroRNA-145 targets TRIM2 and exerts tumor-suppressing functions in epithelial ovarian cancer. Gynecol Oncol. 2015;139(3):513-9.

24. Li J, Zhang S, Wu L, Pei M. Interaction between LncRNA-ROR and miR-145 contributes to epithelialmesenchymal transition of ovarian cancer cells. General physiology and biophysics. 2019;38(6):46171.

25. Hu J, Wang L, Chen J, Gao H, Zhao W, Huang Y, et al. The circular RNA circ-ITCH suppresses ovarian carcinoma progression through targeting miR-145/RASA1 signaling. Biochemical and biophysical 
research communications. 2018;505(1):222-8.

26. Gu S, Cheung HH, Lee TL, Lu G, Poon WS, Chan WY. Molecular mechanisms of regulation and action of microRNA-199a in testicular germ cell tumor and glioblastomas. PloS one. 2013;8(12):e83980.

27. Du L, Qian X, Dai C, Wang L, Huang D, Wang S, et al. Screening the molecular targets of ovarian cancer based on bioinformatics analysis. Tumori. 2015;101(4):384-9.

28. Sumigama S, Ito T, Kajiyama H, Shibata K, Tamakoshi K, Kikkawa F, et al. Suppression of invasion and peritoneal carcinomatosis of ovarian cancer cells by overexpression of AP-2alpha. Oncogene. 2004;23(32):5496-504.

\section{Figures}




\begin{tabular}{lllll}
\hline Case ID & Tumor sample ID & Sample time point & Sample type & Tumor sample purity (qpure) \\
\hline AOCS_093 & ICGCDBPC20130205064 & primary & Primary Tumor & 0.610 \\
AOCS_171 & ICGCDBDE20131003011 & primary & Primary Tumor & 0.805 \\
AOCS_170 & ICGCDBDE20131003008 & primary & Primary Tumor & 0.867 \\
AOCS_093 & ICGCDBDE20131003001 & Primary & Ascites & 0.988 \\
AOCS_170 & ICGCDBDE20131003009 & Primary & Ascites & 0.829 \\
AOCS_171 & ICGCDBDE20131003012 & Primary & Ascites & 0.826 \\
\hline
\end{tabular}

C

miR-199a-3p+miR-199b-3p

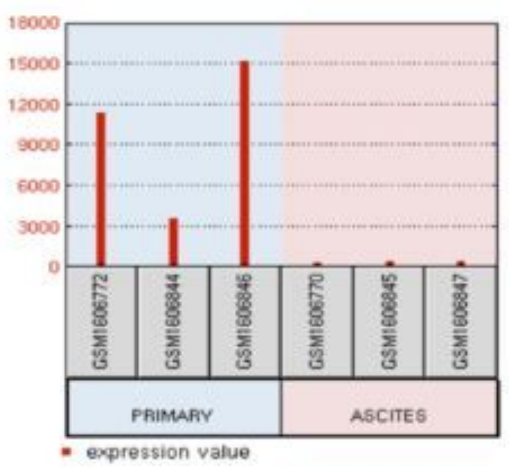

miR-126-3p

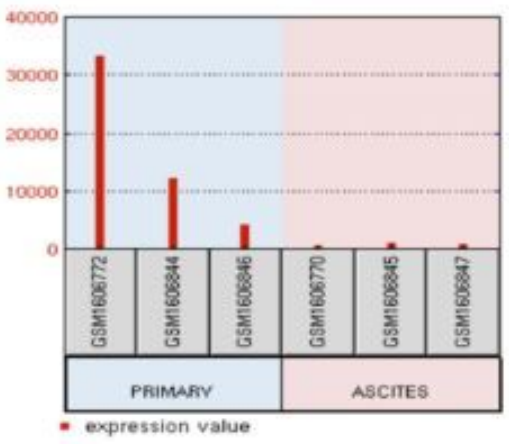

miR-199a-5p

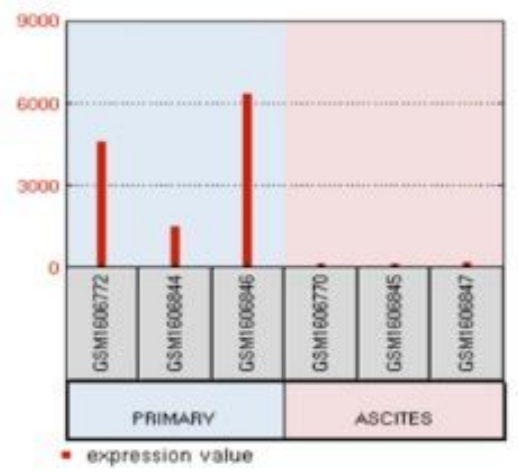

miR-508-5p

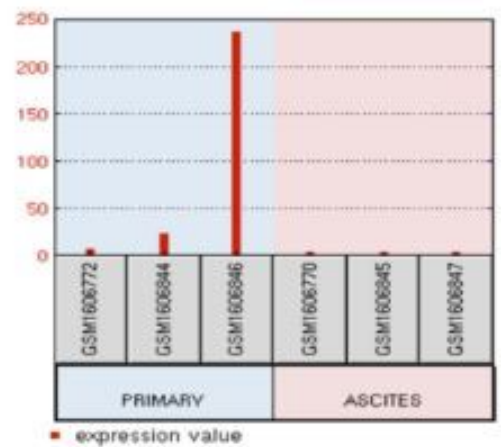

Volcano Plot

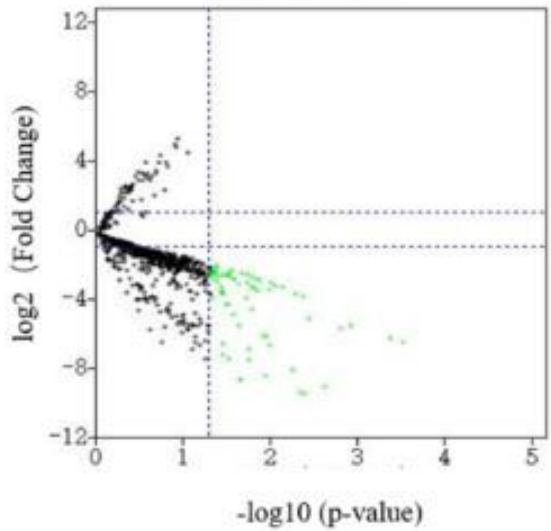

miR-145-5p

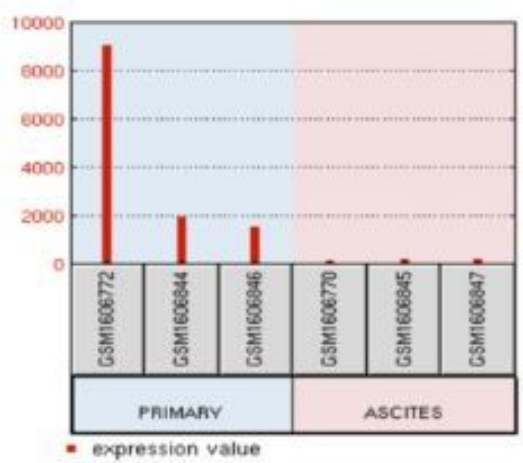

miR-1245a

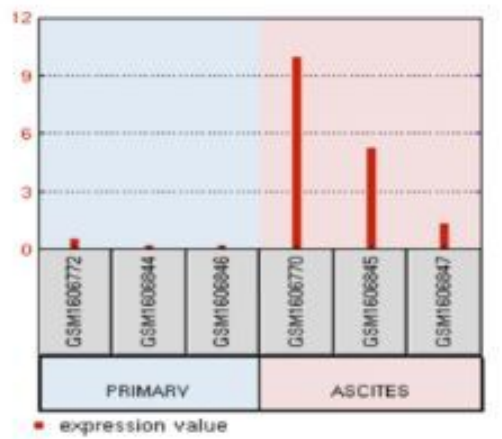

\section{Figure 1}

Identification of DE-miRNAs between ovarian cancer primary tumour tissues and ascites-derived tumour cells. A: Information for the 3 selected HGSOC patients. B: Volcano plot of the DE-miRNAs. The red and green dots represent the upregulated and downregulated DE-miRNAs in ascites-derived ovarian cancer cells, respectively. The black dots represent miRNAs that were not significantly differentially expressed. C: The miRNA expression values of miR-199a-3p, miR-199b-3p, miRNA-199a-5p, miR-145-5p, miR-126-3p, 
miR-508-5p and miR-1245a in ovarian cancer primary tumour tissues and ascites-derived tumour cells of the 3 selected HGSOC patients.

A

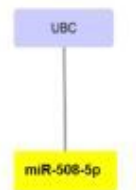

B

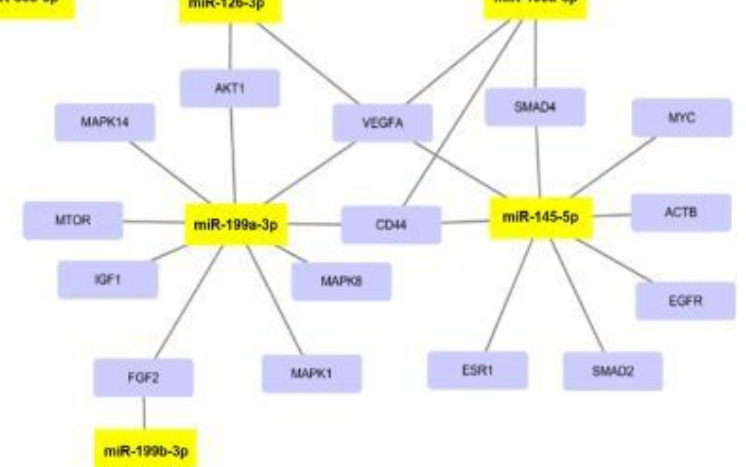

KEGG pathway analysis of target genes of downregulatd miRNAs

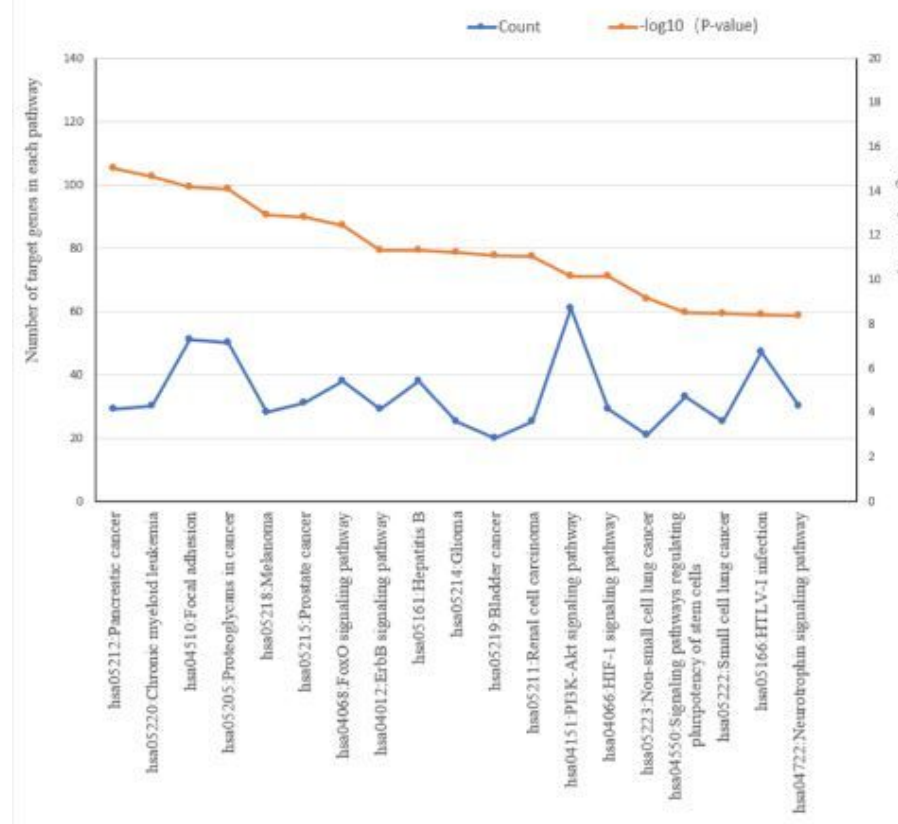

C

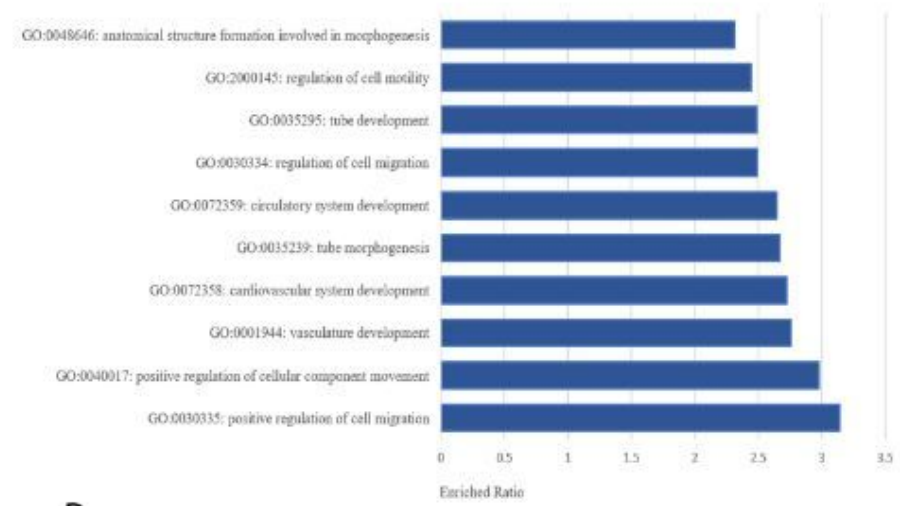

D

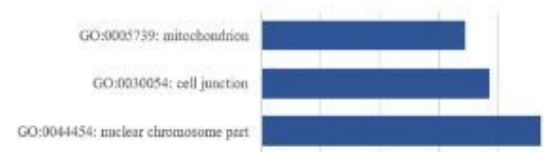

Co:-0070161: ancharing junctice

CO:0000228: mistear cturmosom

corocos912: atherens junctioc

Go 0005925 focal adtession

Co: 0005667: tamsciption facor comples

G03044798: unclear transcaiption factor complex

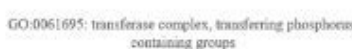

E
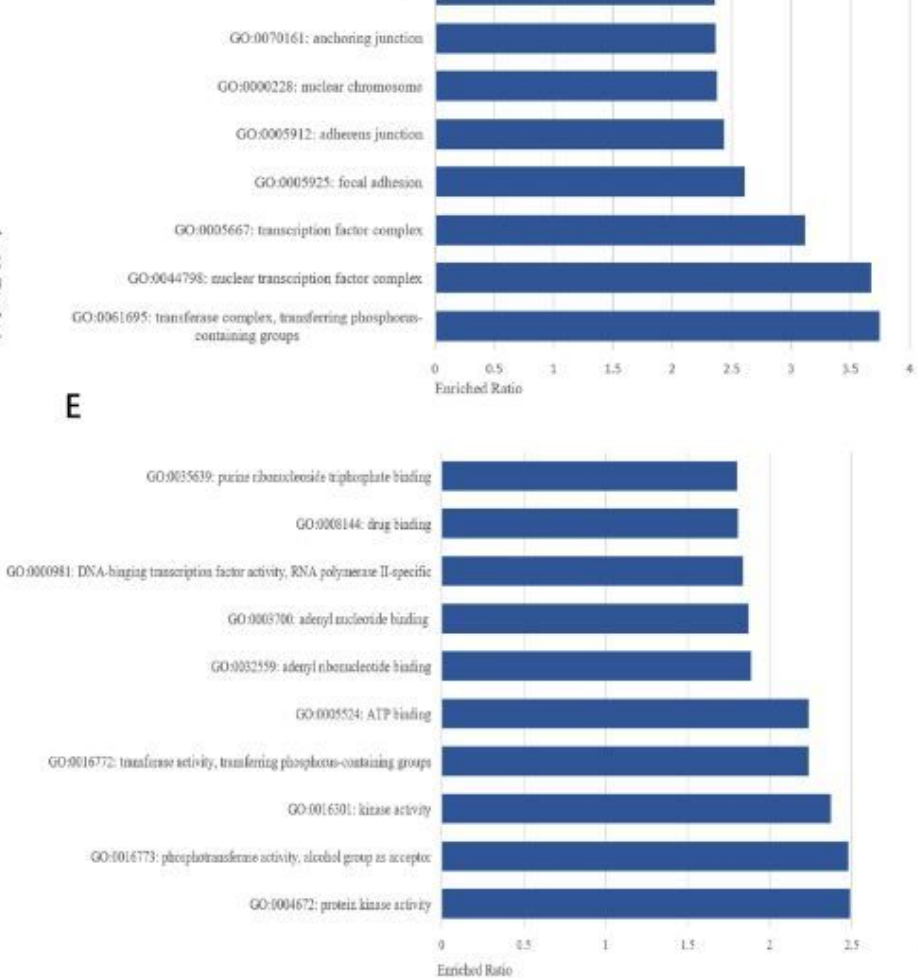

Figure 2

Prediction and functional enrichment of hub target genes of miR-199a-3p, miR-199b-3p, miRNA-199a-5p, miR-145-5p, miR-126-3p. A: The PPI network of hub target genes of the 5 selected DE-miRNAs. B: KEGG pathway analysis of the hub target genes of the 5 selected DE-miRNAs. C-E: GO functions for the hub target genes of the 5 selected DE-miRNAs, including biological process (C), cellular component (D), molecular function (E). 
A

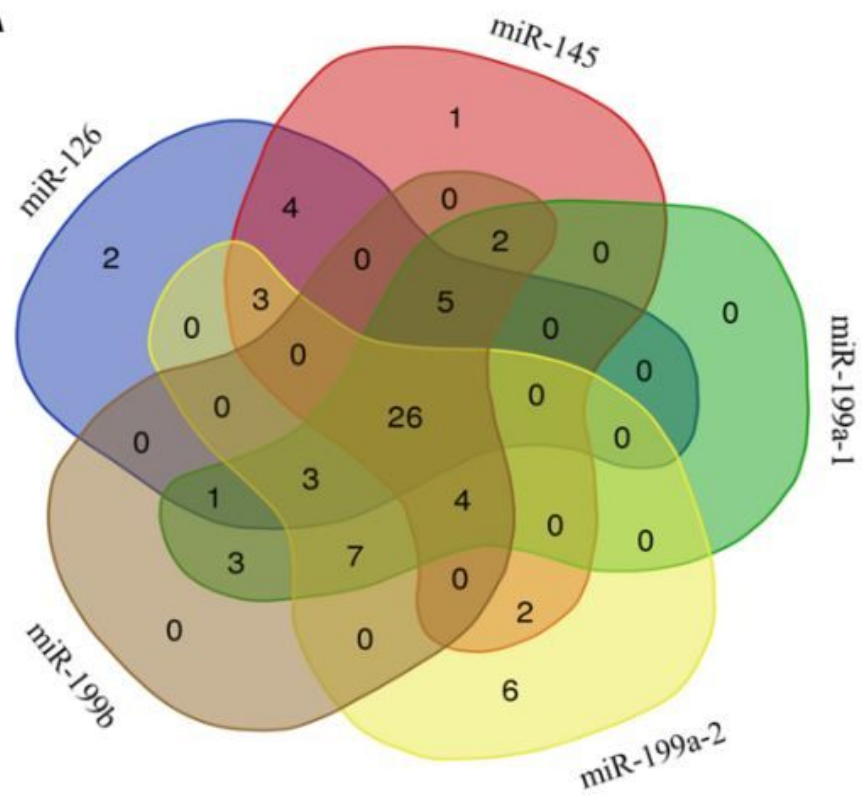

C

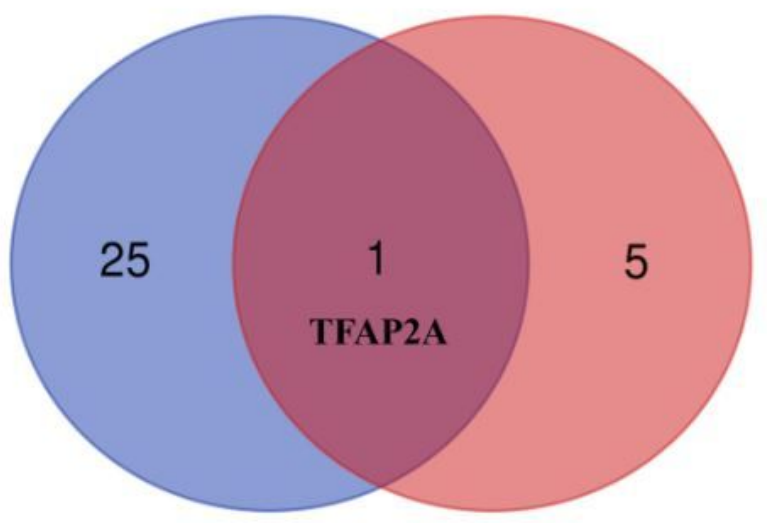

B

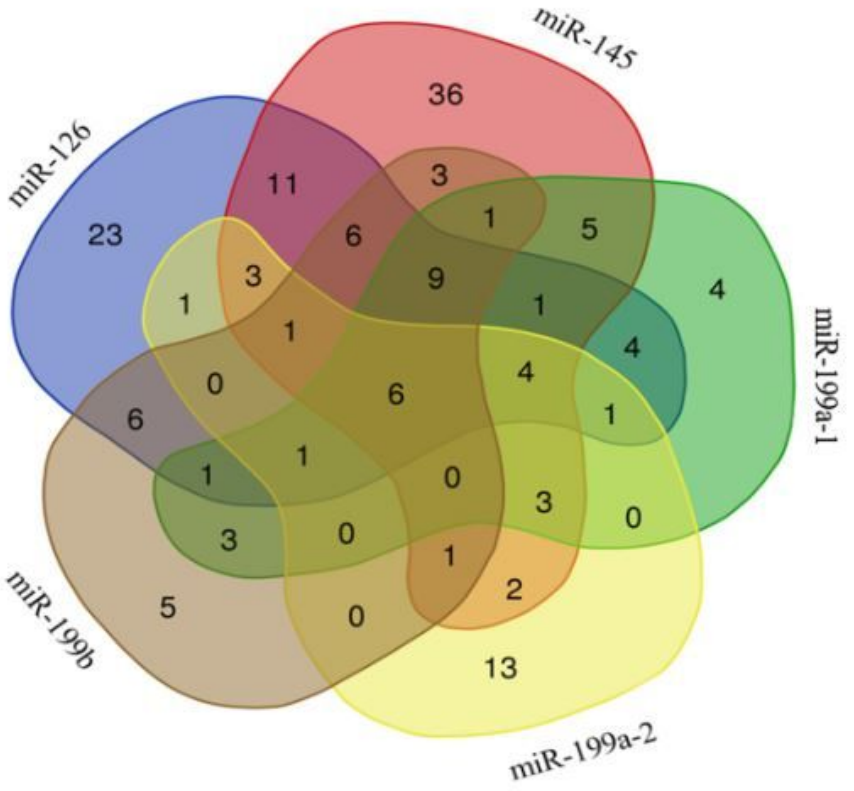

\section{Figure 3}

Identification of potential upstream transcription factors of miR-199a-3p, miR-199b-3p, miRNA-199a-5p, miR-145-5p, miR-126-3p. A: Venn diagram showing 26 common TFs of the 5 selected DE-miRNAs predicted by ALGGEN PROMO. B: Venn diagram showing 6 common TFs of the 5 selected DE-miRNAs predicted by TransmiR v2.0. C: Venn diagram showing that TFAP2A is the common TF of the 5 selected DE-miRNAs after taking the intersection of $A$ and $B$. 
A
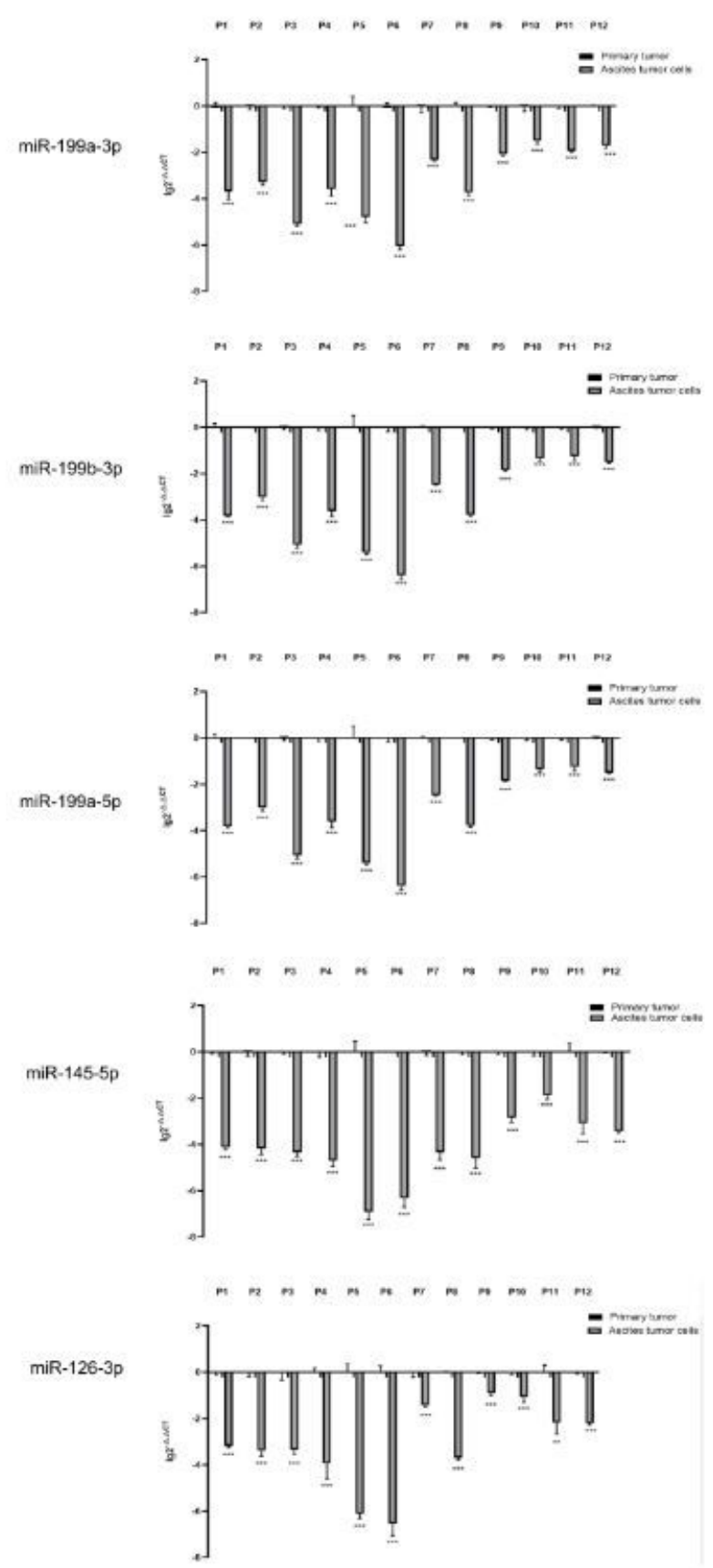

B

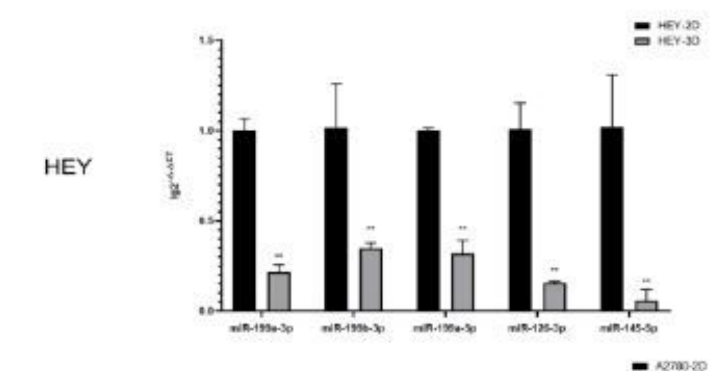

A2780
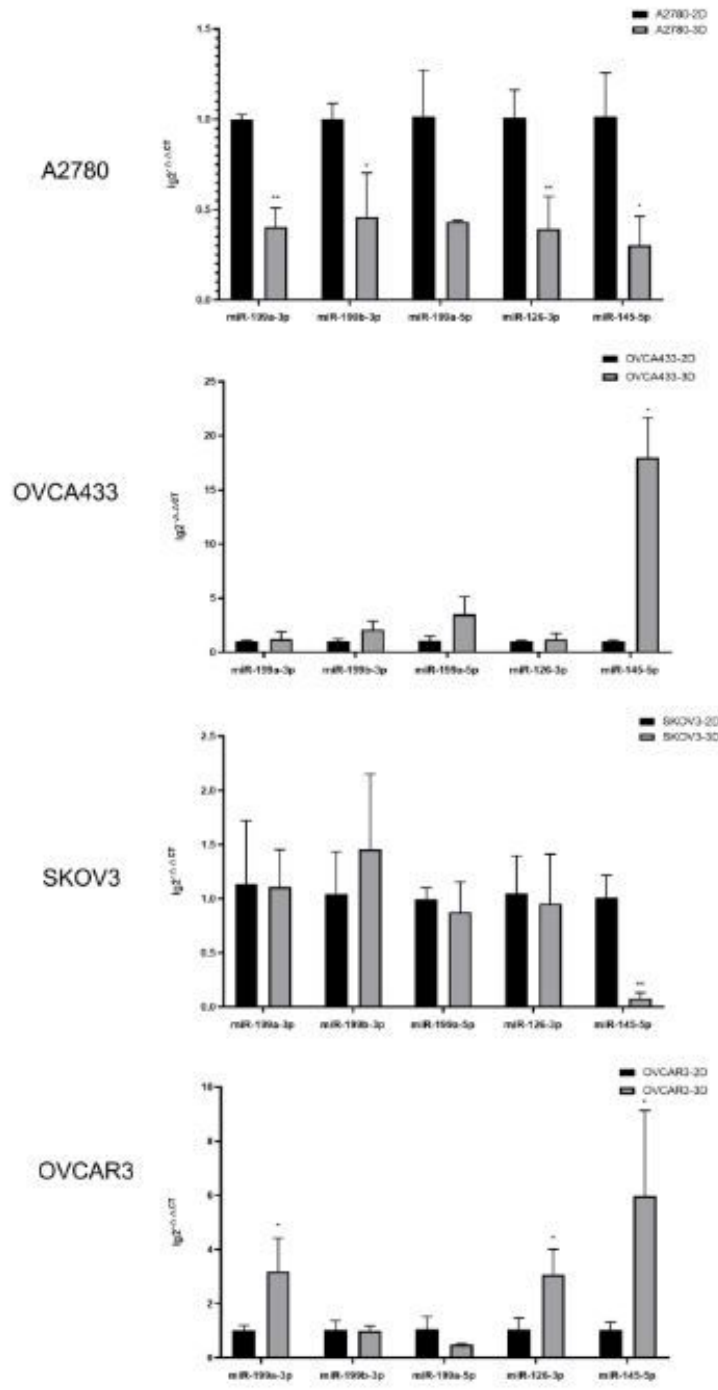

Figure 4

Validation of expression differences of DE-miRNAs between ovarian cancer primary tumours and ascitesderived tumour cells. A: Expression differences of miR-199a-3p, miR-199b-3p, miRNA-199a-5p, miR-145$5 p$, miR-126-3p between 12 pairs of ovarian cancer primary tumour tissues and ascites-derived tumour cells from 12 HGSOC patients via quantitative PCR. B: Expression differences in miR-199a-3p, miR-199b3p, miRNA-199a-5p, miR-145-5p, miR-126-3p expression between adherent cultured (2D) and suspended 
cultured (3D) cells of the ovarian cancer cell lines HEY, A2780, SKOV3, OVCA433 and OVCAR3 via quantitative PCR.

A

A

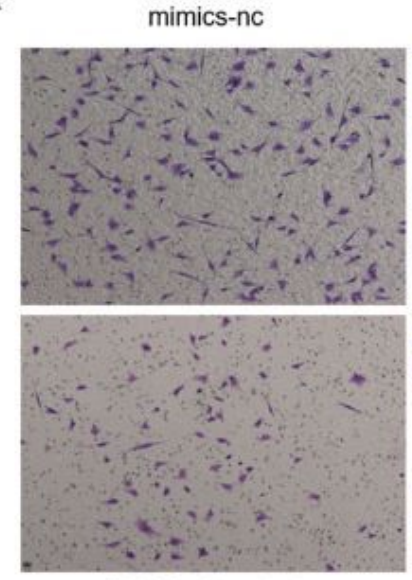

miR-199a-3p mimics
miR-126-3p mimics

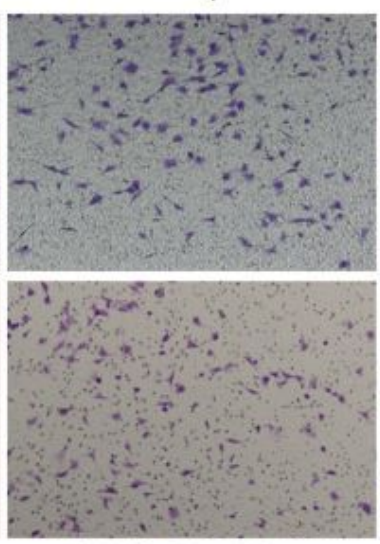

miR-199a-5p mimics
miR-145-5p mimics

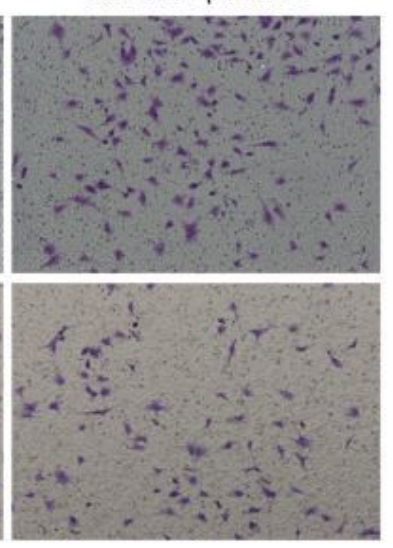

miR-199b-3p mimics

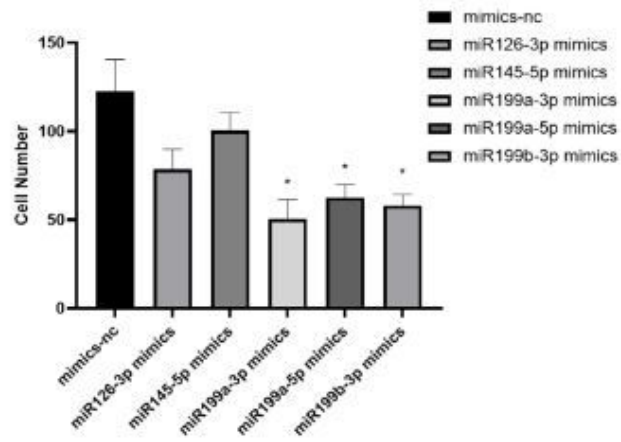

B

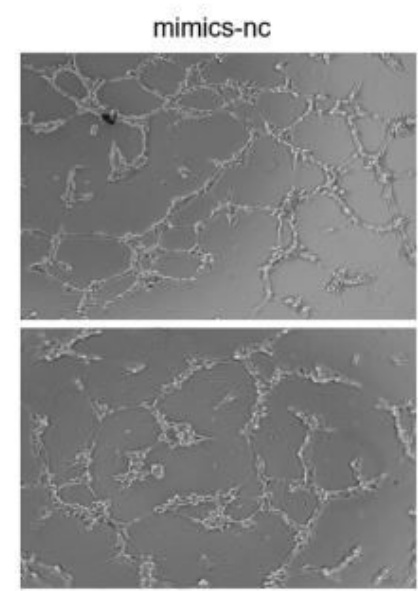

miR-199a-3p mimics
miR-126-3p mimics
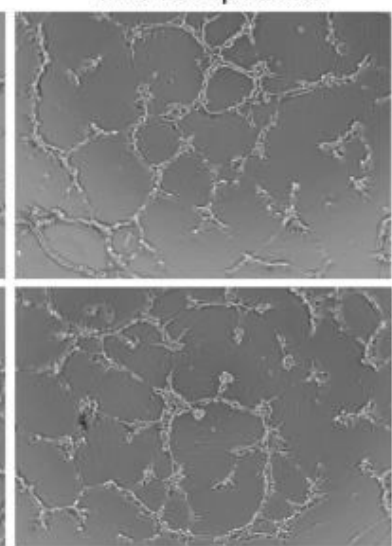

miR-199a-5p mimics
miR-145-5p mimics
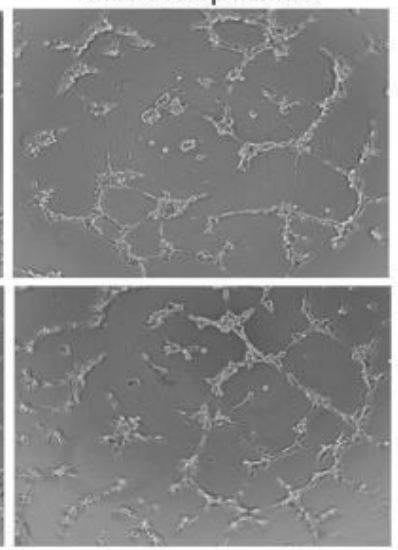

miR-199b-3p mimics

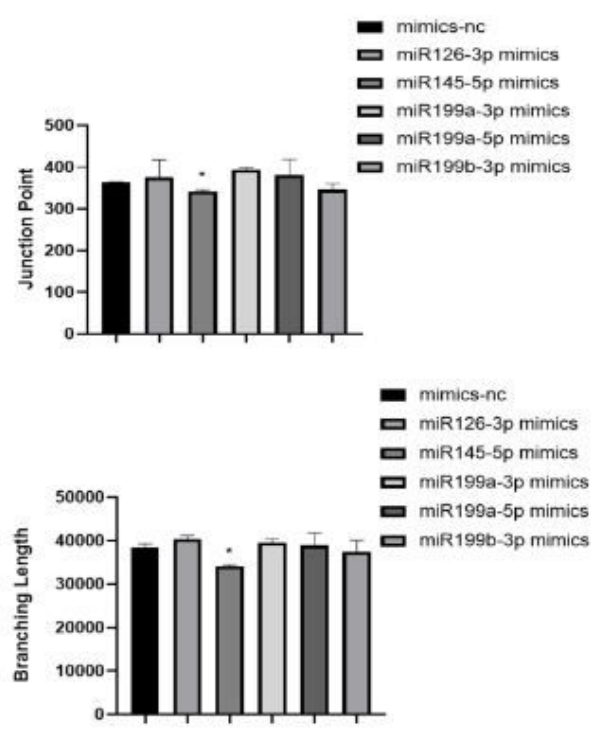

\section{Figure 5}

Effects of miR-199a-3p, miR-199b-3p, miR-199a-5p, miR-145-5p and miRNA-126-3p on invasion and angiogenesis. A: Transwell invasion assay results showing the changes in the invasive ability of 3Dcultured HEY cells after overexpressing miR-199a-3p, miR-199b-3p, miR-199a-5p, miR-145-5p and miRNA126-3p respectively compared with the negative control. B: Angiogenesis assay results showing the changes in the ability to induce HUVEC angiogenesis by 3D-cultured HEY cells overexpressing miR-199a$3 p$, miR-199b-3p, miR-199a-5p, miR-145-5p and miRNA-126-3p compared with negative control. 